\title{
Association of Major Cardiovascular Risk Factors and the Severity of Coronary Artery Disease with Vitamin D Level
}

\author{
Ahmed Hussein ${ }^{1, *}$, Sherif Abdelaziz Sayed ${ }^{2}$, Mohammad Shafiq Awad ${ }^{3}$ \\ ${ }^{1}$ Department of Internal Medicine, Faculty of Medicine, Sohag University, Sohag, Egypt \\ ${ }^{2}$ Department of Clinical Pathology, Faculty of Medicine, Sohag University, Sohag, Egypt \\ ${ }^{3}$ Department of Cardiology, Faculty of Medicine, Beni Suef University, Beni Suef, Egypt \\ Email address: \\ forahmedaly@yahoo.com (A. Hussein), shereefaziz_2@yahoo.com (S. A. Sayed), medo_dode123@yahoo.com (M. S. Awad) \\ ${ }^{*}$ Corresponding author

\section{To cite this article:} \\ Ahmed Hussein, Sherif Abdelaziz Sayed, Mohammad Shafiq Awad. Association of Major Cardiovascular Risk Factors and the Severity of \\ Coronary Artery Disease with Vitamin D Level. Cardiology and Cardiovascular Research. Vol. 4, No. 3, 2020, pp. 119-126. \\ doi: 10.11648/j.ccr.20200403.17
}

Received: July 25, 2020; Accepted: August 5, 2020; Published: August 13, 2020

\begin{abstract}
Objectives: We aimed to investigate the association of major cardiovascular risk factors and the severity of coronary artery disease (CAD) with vitamin D level. Background: Numerous efforts have been made to reduce cardiovascular morbidity and mortality, especially in acute coronary syndrome (ACS). However, results are still not optimal. Much consideration is set on the revelation of new, conceivably modifiable cardiovascular risk factors. Vitamin D deficiency is proposed to be one such factor, and it might be related to an increased risk of cardiovascular diseases. Methods: We conducted a cross-section study on 475 patients undergoing elective coronary angiography, the participants were subjected to assessment of vitamin $\mathrm{D}$ level and major cardiovascular risk factors. The severity of CAD was assessed using the gensini score. Results: There were 352 (74.11\%) patients, had a significant CAD. We found that vitamin D level had a significant negative correlation with the gensini score while diabetes and dyslipidemia had a significant positive correlation. The multivariate regression analysis showed that, vitamin D deficiency, diabetes, dyslipidemia, higher BMI, and ACS (p-value $<0.05$ ), were statistically significant predictors of a significant CAD. There were 184 (38.74\%) patients, had vitamin D deficiency. Also, we found that diabetes and dyslipidemia had a significant negative correlation with vitamin D level. The multivariate regression analysis showed that diabetes and dyslipidemia were statistically significantly associated with low vitamin D levels (p-value 0.005 and 0.024 respectively). Conclusions: Vitamin D deficiency, diabetes, dyslipidemia, higher BMI, and ACS were statistically significant associated with severe CAD. Also, diabetes and dyslipidemia were statistically significant associated with vitamin D deficiency.
\end{abstract}

Keywords: CAD, Cardiovascular Risk Factors, Gensini Score, Vitamin D Level

\section{Introduction}

CAD is one of the leading causes of death worldwide. Numerous efforts have been made to reduce cardiovascular morbidity and mortality, especially in ACS. However, results are still not optimal [1-4]. Much consideration is set on the revelation of new, conceivably modifiable cardiovascular risk factors. Vitamin D deficiency is proposed to be one such factor, and it might be related to an increased risk of cardiovascular diseases [5-7]. Vitamin D is a prohormone with numerous consequences on the immune system, inflammatory response, and coagulative and thrombotic pathways, therefore connecting vitamin $\mathrm{D}$ to the pathogenesis of CAD, and with the occurrence of its acute complications $[2,3,8,9]$. Additionally, it has been shown that a deficient vitamin D level may influence the cardiovascular system through a classic mechanism, to be specific by advancing changes in the calcium-phosphate metabolism, or through impacts on the renin-angiotensin-aldosterone system [10]. The impacts of vitamin D deficiency were seen at all stages of atherosclerotic plaque formation, destabilization, and rupture [11]. Besides, it has previously been shown that lower levels of vitamin D are independently connected with 
other cardiovascular risk factors, for example, hypertension, diabetes, obesity, and metabolic syndrome [12-15]. In contrast, in a study conducted on a heterogeneous Polish population of cardiac patients with hypertension, diabetes, and/or dyslipidemia, there was no significant association between the level of the $25(\mathrm{OH}) \mathrm{D}$ and the stage of coronary atherosclerosis [16]. So, we aimed to investigate the association of major cardiovascular risk factors and the severity of CAD with vitamin D level.

\section{Materials and Methods}

\subsection{Design of the Study}

This was a cross-section observational two-center study conducted on 475 patients $\geq 18$ years, presented with stable CAD or ACS indicated for elective coronary angiography, at Sohag and Beni Suef university hospitals.

All subjects provided written informed consent to participate in the study. The study protocol was approved by the ethics committee at Sohag and Beni Suef Faculty of medicine.

Patients were subjected to:

1 Identification of major cardiovascular risk factors (age, gender, smoking, hypertension, diabetes mellitus, dyslipidemia, body mass index (BMI), and Family history of CAD).

2 Assessment of symptoms suggestive of coronary insufficiency as typical exertional chest pain or history of admission to the coronary care unit with ACS.

3 Laboratory testing includes, vitamin D assay, fasting blood sugar, hemoglobin Alc level, lipid profile, liver function, and renal function tests.

4 Assessment of vitamin D level: Vitamin D (25-OH D3) assay was done by using MINI VIDAS ${ }^{\circledR}$, bioMérieux which is a compact automated immunoassay system based on the Enzyme-Linked Fluorescent Assay (ELFA) principles. ELFA combine the methodology of ELISA testing with the utilization of a fluorescent label in the final detection process, Due to the use of combined labels, the results yield a high level of sensitivity and specificity with rapid turnaround times. The normal value for 25-OH D3 levels in our laboratory is $\geq 30 \mathrm{ng} / \mathrm{ml}$, vitamin D insufficiency was from 20 to $30 \mathrm{ng} / \mathrm{ml}$, vitamin $\mathrm{D}$ deficiency was considered for values $<20 \mathrm{ng} / \mathrm{ml}$, and severe vitamin D deficiency was considered for values $<10 \mathrm{ng} / \mathrm{ml}$. [17]

5 Coronary angiography was performed by an experienced interventional cardiologist who was blinded for the patient characteristics. Assessment of the severity of CAD had been done by using Gensini score, Gensini score grades narrowing of the lumen of the coronary artery and scores it with numerical values as the following: 1 for $1-25 \%$ narrowing, 2 for $26-50 \%$ narrowing, 4 for $51-75 \%$, and 8 for $76-90 \%, 16$ for $91-99 \%$, and 32 for a totally occluded artery. This score is then multiplied by a factor, according to the site of lesion: The multiplication factor is 5 for a left main stem lesion, 2.5 for proximal left anterior descending artery (LAD) and proximal circumflex artery $(\mathrm{CX})$ lesions, 1.5 for a mid-LAD lesion, 1 for distal $\mathrm{LAD}$, mid/distal $\mathrm{CX}$ and right coronary artery lesions and 0.5 for any other branch. They were further divided into mild CAD group with Gensini score $<26$, moderate CAD group with Gensini score being 26-54 and severe CAD group with Gensini score $>54$ (the patient was considered to have a significant $\mathrm{CAD}$ if the gensini score was $\geq 26)$. [18]

Exclusion criteria:

Patients receiving vitamin D or calcium supplementation, advanced liver disease, chronic kidney disease (stage III-V), patients had cancer and patients with fever or elevated inflammatory markers.

Definitions:

In this study, the participant was considered diagnosed diabetic if (1) other than during pregnancy he had a physician previously told him that he has diabetes, or (2) taking antidiabetic treatment. Also, participants were considered as newly diagnosed diabetics if they had one of the following: 1- Fasting plasma glucose $\geq 126 \mathrm{mg} / \mathrm{dl} \mathrm{2-} \mathrm{Symptoms} \mathrm{of}$ hyperglycemia and random plasma glucose $\geq 200 \mathrm{mg} / \mathrm{dl} 3$ HbAlc $\geq 6.5 \%$ [19].

Systemic hypertension was defined, as a blood pressure $\geq 140$ / $\geq 90 \mathrm{mmHg}$ and/or on current antihypertensive therapy [20].

Elevated triglyceride was defined as triglyceride of $\geq 150$ $\mathrm{mg} / \mathrm{dl}$. Low HDL cholesterol was defined as $<40 \mathrm{mg} / \mathrm{dl}$ in males and $<50 \mathrm{mg} / \mathrm{dl}$ in females. Elevated LDL was defined as $100 \mathrm{mg} / \mathrm{dl}$ or greater [21].

The following formula was used to calculate the minimum size of the required sample:

$$
\mathrm{n}=(\mathrm{z})^{2} \mathrm{p}(1-\mathrm{p}) / \mathrm{d}^{2}
$$

where $\mathrm{n}$ indicates the sample size, $\mathrm{z}$ indicates the level of confidence according to the standard normal distribution (for a level of confidence of $95 \%, z=1.96), p$ indicates the estimated proportion of the population that presents the characteristic, $d$ indicates the tolerated margin of error (for example, we want to know the real proportion within 5\%).

Using the previous formula for the sample size calculation $(\mathrm{n})=(1.96)^{2} \times 0.5(1-0.5) /(0.05)^{2}=384.16$. So, the minimum sample size is 385 participants.

\subsection{Analysis of Data}

Data were analyzed using SPSS version 19. Quantitative data were analyzed using the analysis of variance (ANOVA) to compare the means of three or more groups. Qualitative data were compared using the Chi-square test. Fisher's exact correction was used when the expected cell count is less than five. Pearson correlation was used to study the correlation between the Gensini score and other variables and the correlation between vitamin D level and major cardiovascular risk factors. Univariate and multivariate regression analyses were done. Odds ratio (OR) at 95\% confidence interval $(\mathrm{CI})$ and $\mathrm{p}$ values were computed. Pvalue was considered significant at or below 0.05 . 


\section{Results}

The study was conducted on 475 patients $\geq 18$ years old, a candidate for elective coronary angiography. Table 1 shows the assessment of the severity of CAD using the gensini score and vitamin D level.

There were $352(74.11 \%)$ out of 475 patients, had a significant CAD. We found that, vitamin D level had a significant negative correlation with gensini score (correlation coefficient ' $r$ ' $=-0.430$, $p$ value $<0.001$ ), while diabetes and dyslipidemia had a significant positive correlation (' $r$ ' $=0.299$ and 220 respectively, $p$ value $<0.001$ ) (Table 2).

The multivariate regression analysis showed that, vitamin D deficiency, diabetes, dyslipidemia, higher BMI, and patients presented with ACS were statistically significant associated with severe CAD (OR: 2.134, 95\% CI: $1.637-$ 2.781; p-value <0.001), (OR: 2.890, 95\% CI: 1.789-4.670; pvalue $<0.001$ ), (OR: 3.639 , 95\% CI: 2.083-6.358; p-value $<0.001$ ), (OR: 1.089, 95\% CI: 1.013-1.172; p-value 0.021) and (OR: 1.641, 95\% CI: 1.138-2.367; p-value 0.008) respectively. (Tables 3,4 )

There were $184(38.74 \%)$ patients had vitamin D deficiency. We found that, diabetes and dyslipidemia had a significant negative correlation with vitamin D level $(' r '=-$ 0.169 , p-value $<0.001$ and ' $\mathrm{r}$ '=- 0.141 , p-value 0.002 respectively). (Table 5)

The multivariate regression analysis showed that diabetes and dyslipidemia were statistically significant associated with vitamin D deficiency (OR: $1.757,95 \% \mathrm{CI}: 1.180-2.614, \mathrm{p}-$ value 0.005 and OR: $1.665,95 \% \mathrm{CI}: 1.069-2.594$, p-value 0.024 respectively) (Tables 6,7 ).

Table 1. Distribution of patient characteristics in relation to gensini score.

\begin{tabular}{|c|c|c|c|c|}
\hline \multirow{3}{*}{ Characteristic } & \multicolumn{3}{|l|}{ Gensini Score } & \multirow{3}{*}{ P value } \\
\hline & $<26$ & -54 & $>54$ & \\
\hline & $\mathrm{N}=123(25.89 \%)$ & $\mathrm{N}=188(39.58 \%)$ & $\mathrm{N}=164(34.53 \%)$ & \\
\hline \multicolumn{4}{|l|}{ Age (years) } & \multirow{2}{*}{0.18} \\
\hline Mean \pm SD & $50.52 \pm 9.35$ & $52.74 \pm 10.54$ & $51.49 \pm 11.27$ & \\
\hline \multicolumn{5}{|l|}{ Gender } \\
\hline Male & $85(69.11 \%)$ & $132(70.21 \%)$ & $104(63.41 \%)$ & \multirow[t]{2}{*}{0.363} \\
\hline Female & $38(30.89 \%)$ & $56(29.79 \%)$ & $60(36.59 \%)$ & \\
\hline \multicolumn{4}{|l|}{ Hypertension } & \multirow{2}{*}{0.258} \\
\hline Yes & $57(46.34 \%)$ & $99(52.66 \%)$ & $92(56.10 \%)$ & \\
\hline \multicolumn{4}{|l|}{ Diabetes } & \multirow{2}{*}{$<0.001$} \\
\hline Yes & $45(36.59 \%)$ & $108(57.45 \%)$ & $123(75 \%)$ & \\
\hline \multicolumn{4}{|l|}{ Dyslipidemia } & \multirow{2}{*}{$<0.001$} \\
\hline Yes & $36(29.27 \%)$ & $107(56.91 \%)$ & $97(59.15 \%)$ & \\
\hline \multicolumn{4}{|l|}{ Smoking } & \multirow{2}{*}{0.293} \\
\hline Yes & $63(51.22 \%)$ & $100(53.19 \%)$ & $98(59.76 \%)$ & \\
\hline Mean \pm SD & $26.20 \pm 3.34$ & $27.07 \pm 3.64$ & $26.88 \pm 3.33$ & 0.082 \\
\hline \multicolumn{4}{|l|}{ Family history } & \multirow{2}{*}{0.937} \\
\hline Yes & $22(17.89 \%)$ & $31(16.49 \%)$ & $27(16.46 \%)$ & \\
\hline \multicolumn{5}{|c|}{ Clinical presentation } \\
\hline Stable CAD & $23(18.70 \%)$ & $23(12.23 \%)$ & $18(10.98 \%)$ & \multirow[t]{2}{*}{0.350} \\
\hline ACS & $100(81.30 \%)$ & $165(87.77 \%)$ & $146(89.02 \%)$ & \\
\hline \multicolumn{4}{|l|}{ Vitamin D level } & \multirow{5}{*}{$<0.001$} \\
\hline$<10$ & $4(3.25 \%)$ & $15(7.98 \%)$ & $30(18.29 \%)$ & \\
\hline$\geq 10-<20$ & $11(8.94 \%)$ & $52(27.66 \%)$ & $72(43.90 \%)$ & \\
\hline$\geq 20-<30$ & $35(28.46 \%)$ & $54(28.72 \%)$ & $32(19.51 \%)$ & \\
\hline$\geq 30$ & $73(59.35 \%)$ & $67(35.64 \%)$ & $30(18.29 \%)$ & \\
\hline
\end{tabular}

Abbreviations: ACS, acute coronary syndrome: BMI, body mass index: CAD, coronary artery disease: N, number: SD, standard deviation.

Table 2. Correlation between gensini score and other variables.

\begin{tabular}{lll}
\hline Variables & Correlation coefficient (r) & P value \\
\hline Age & 0.028 & 0.539 \\
Gender (male/ female) & 0.051 & 0.27 \\
Hypertension & 0.074 & 0.106 \\
Diabetes & 0.299 & $<0.001$ \\
Dyslipidemia & 0.220 & $<0.001$ \\
Smoking & 0.068 & 0.137 \\
BMI & 0.070 & 0.128 \\
Family history & 0.014 & 0.763 \\
Clinical presentation Stable CAD ACS & 0.082 & 0.075 \\
Vitamin D level & -0.430 & $<0.001$ \\
\hline
\end{tabular}

Abbreviations: ACS, acute coronary syndrome: BMI, body mass index: CAD, coronary artery disease: r, correlation coefficient. 
Table 3. Univariate analysis of variables as regards to severity of CAD.

\begin{tabular}{|c|c|c|c|}
\hline \multirow{2}{*}{ Variable } & \multirow{2}{*}{$\begin{array}{l}\text { Gensini score < 26 } \\
\mathrm{N}=123(25.89 \%) \\
\end{array}$} & \multirow{2}{*}{$\begin{array}{l}\text { Gensini score } \geq 26 \\
\mathrm{~N}=352(74.11 \%) \\
\end{array}$} & \multirow{2}{*}{$P$ value } \\
\hline & & & \\
\hline \multicolumn{3}{|l|}{ Age (years) } & \multirow{2}{*}{0.138} \\
\hline Mean \pm SD & $50.52 \pm 9.35$ & $52.16 \pm 10.89$ & \\
\hline \multicolumn{4}{|l|}{ Gender } \\
\hline Male & $85(69.11 \%)$ & $236(67.05 \%)$ & \multirow[t]{2}{*}{0.674} \\
\hline Female & $38(30.89 \%)$ & $116(32.95 \%)$ & \\
\hline \multicolumn{3}{|l|}{ Hypertension } & \multirow{2}{*}{0.130} \\
\hline Yes & $57(46.34 \%)$ & $191(54.26 \%)$ & \\
\hline \multicolumn{3}{|l|}{ Diabetes } & \multirow{2}{*}{$<0.001$} \\
\hline Yes & $45(36.59 \%)$ & $231(65.63 \%)$ & \\
\hline \multicolumn{3}{|l|}{ Dyslipidemia } & \multirow{2}{*}{$<0.001$} \\
\hline Yes & $36(29.27 \%)$ & $204(57.95 \%)$ & \\
\hline \multicolumn{3}{|l|}{ Smoking } & \multirow{2}{*}{0.334} \\
\hline Yes & $63(51.22 \%)$ & $198(56.25 \%)$ & \\
\hline \multicolumn{3}{|l|}{$\operatorname{BMI}\left(\mathrm{kg} / \mathrm{m}^{2}\right)$} & \multirow{2}{*}{0.029} \\
\hline Mean \pm SD & $26.20 \pm 3.34$ & $26.99 \pm 3.49$ & \\
\hline \multicolumn{3}{|l|}{ Family history } & \multirow{2}{*}{0.719} \\
\hline Yes & $22(17.89 \%)$ & $58(16.49 \%)$ & \\
\hline \multicolumn{4}{|c|}{ Clinical presentation } \\
\hline Stable CAD & $23(18.70 \%)$ & $41(11.65 \%)$ & \multirow[t]{2}{*}{0.125} \\
\hline ACS & $100(81.30 \%)$ & $311(88.35 \%)$ & \\
\hline \multicolumn{3}{|l|}{ Vitamin D level } & \multirow{5}{*}{$<0.001$} \\
\hline$<10$ & $4(3.25 \%)$ & $45(12.78 \%)$ & \\
\hline$\geq 10-<20$ & $11(8.94 \%)$ & $124(35.23 \%)$ & \\
\hline$\geq 20-<30$ & $35(28.46 \%)$ & $86(24.43 \%)$ & \\
\hline$\geq 30$ & $73(59.35 \%)$ & $97(27.56 \%)$ & \\
\hline
\end{tabular}

Abbreviations: ACS, acute coronary syndrome: BMI, body mass index: CAD, coronary artery disease: N, number: SD, standard deviation.

Table 4. Multivariate regression analysis for predictors of severe CAD.

\begin{tabular}{lll}
\hline Variables & OR $\mathbf{( 9 5 \%} \mathbf{C I})$ & P value \\
\hline Age & $0.984(0.961-1.008)$ & 0.193 \\
Gender (male/ female) & $1.053(0.631-1.757)$ & 0.844 \\
Hypertension & $1.377(0.847-2.237)$ & 0.197 \\
Diabetes & $2.890(1.789-4.670)$ & $<0.001$ \\
Dyslipidemia & $3.639(2.083-6.358)$ & $<0.001$ \\
Smoking & $0.679(0.392-1.174)$ & 0.166 \\
BMI & $1.089(1.013-1.172)$ & 0.021 \\
Family history & $1.123(0.593-2.125)$ & 0.721 \\
Clinical presentation ACS /Stable CAD & $1.641(1.138-2.367)$ & 0.008 \\
Vitamin D level & $-2.134(-1.637--2.781)$ & $<0.001$ \\
\hline
\end{tabular}

Abbreviations: ACS, acute coronary syndrome: BMI, body mass index: CAD, coronary artery disease: CI, confidence interval: OR, odds ratio.

Table 5. Correlation between patient characteristics and vitamin D level.

\begin{tabular}{lll}
\hline Variables & Correlation coefficient $(\mathbf{r})$ & P value \\
\hline Age & -0.050 & 0.280 \\
Gender (male/ female) & 0.078 & 0.091 \\
Hypertension & -0.079 & 0.085 \\
Diabetes & -0.169 & $<0.001$ \\
Dyslipidemia & -0.141 & 0.002 \\
Smoking & -0.022 & 0.632 \\
BMI & -0.057 & 0.219 \\
Family history & -0.007 & 0.847 \\
Clinical presentation Stable CAD» ACS & -0.006 & 0.900 \\
\hline
\end{tabular}

Abbreviations: ACS, acute coronary syndrome: BMI, body mass index: CAD, coronary artery disease: r, correlation coefficient. 
Table 6. Distribution of patient characteristics in relation to vitamin D level.

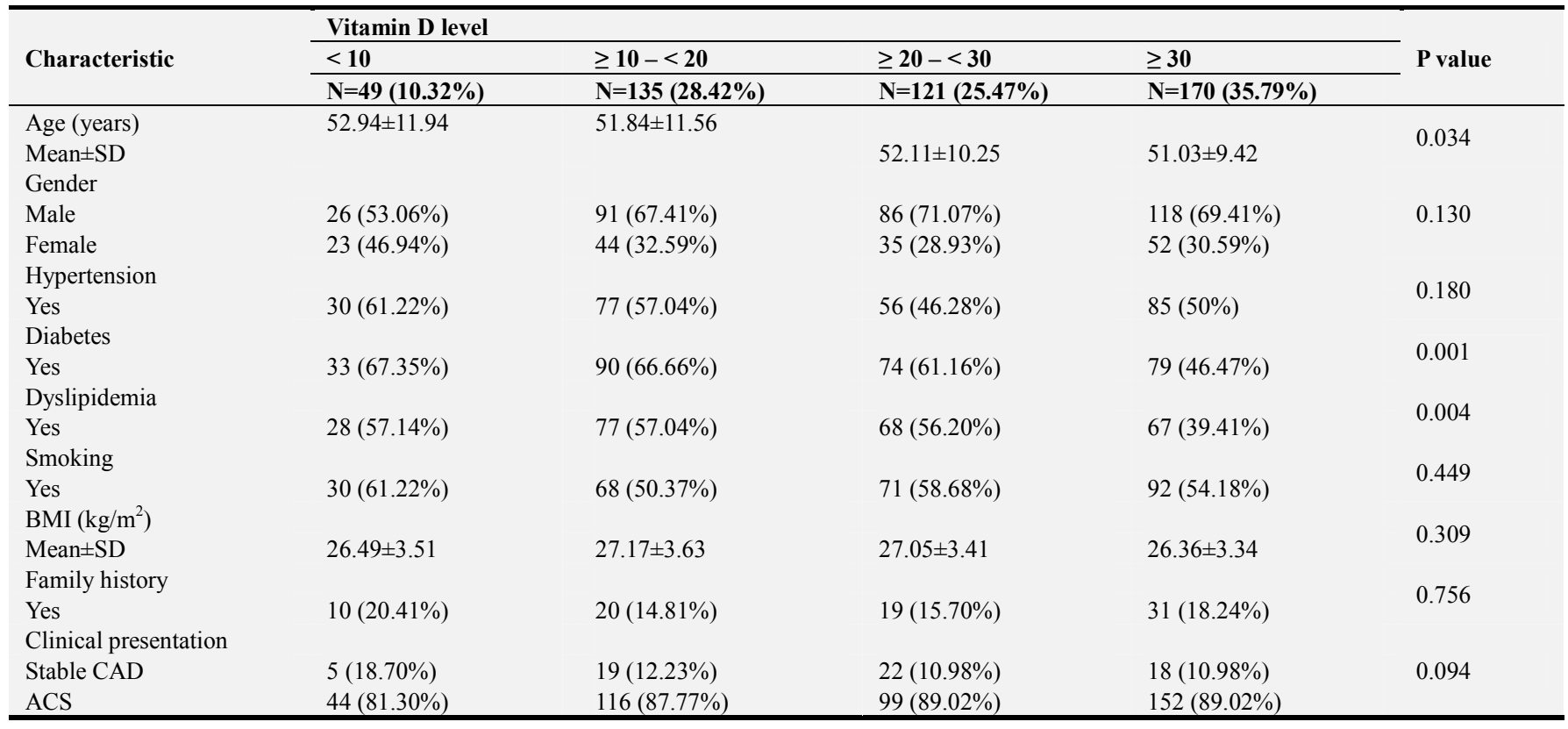

Abbreviations: ACS, acute coronary syndrome: BMI, body mass index: CAD, coronary artery disease: N, number: SD, standard deviation.

Table 7. Multivariate regression analysis for predictors of vitamin D level.

\begin{tabular}{lll}
\hline Variables & OR (95\% CI) & P value \\
\hline Age & $0.994(0.977-1.012)$ & 0.534 \\
Gender (male/ female) & $0.761(0.508-1.141)$ & 0.187 \\
Hypertension & $1.405(0.953-2.071)$ & 0.086 \\
Diabetes & $1.757(1.180-2.614)$ & 0.005 \\
Dyslipidemia & $1.665(1.069-2.594)$ & 0.024 \\
Smoking & $0.677(0.432-1.060)$ & 0.088 \\
BMI & $0.964(0.912-1.018)$ & 0.189 \\
Family history & $0.975(0.572-1.662)$ & 0.927 \\
Clinical presentation Stable CAD» ACS & $0.822(0.613-1.102)$ & 0.822 \\
\hline
\end{tabular}

Abbreviations: ACS, acute coronary syndrome: BMI, body mass index: CAD, coronary artery disease: CI, confidence interval: OR, odds ratio.

\section{Discussion}

Egypt is one of the developing countries, facing a major socio-economic burden regarding the management of the prevalent cardiovascular diseases and their outcomes. So, it is important to detect and manage any well-established preventable and modifiable cardiovascular risk factors and the revelation of new modifiable risk factors to reduce cardiovascular morbidity and mortality.

In our study, we found that $74.11 \%$ of patients, had severe $\mathrm{CAD}$ and $38.74 \%$ of patients had vitamin D deficiency. Also, we identified that vitamin D deficiency, diabetes, dyslipidemia, higher BMI, and ACS were statistically significantly associated with severe CAD. Besides, diabetes and dyslipidemia were statistically significantly associated with vitamin D deficiency. Also, BMI showed a significant positive correlation with the severity of $\mathrm{CAD}$, while vitamin $\mathrm{D}$ level showed a significant negative correlation. Considering the major cardiovascular risk factors, both diabetes and dyslipidemia showed a significant negative correlation with vitamin D level.

Our results were in line with the results of two prospective studies conducted by Dziedzic EA, et al. the first one published in 2017, was conducted on 337 Polish cardiac patients with type 2 diabetes undergoing coronary angiography and showed that, diabetic cardiac patients had significant differences in the vitamin D level between the groups related to the severity of coronary atherosclerosis, and showed that, a group of cardiac patients with diabetes and significant stenosis in three coronary arteries, hospitalized due to ACS, with the history of previous myocardial infarction and hyperlipidemia, had the lowest vitamin $\mathrm{D}$ level. Another study published in 2019 was conducted on 410 nondiabetic patients candidate for coronary angiography reported that, $76 \%$ of patients had vitamin D deficiency and patients with one to three-vessel atherosclerosis have a significantly lower vitamin $\mathrm{D}$ level compared to patients with insignificant $\mathrm{CAD}$, and lower vitamin $\mathrm{D}$ level was observed in ACS patients compared to patients diagnosed with stable CAD. We found a lower incidence of vitamin D deficiency (38.74\%) which could be explained by our sunny climate [22, 23].

Verdoia $\mathrm{M}$, et al. conducted a cross-sectional study on 1484 patients undergoing elective coronary angiography. They found that hypovitaminosis D was observed in $70.4 \%$ 
of patients and vitamin D deficiency was significantly associated with the prevalence and extent of CAD and detected a significant association between vitamin D deficiency and major cardiovascular risk factors such as age, female gender, renal failure, hypercholesterolemia, anemia and smoking, that is matched with our results [24].

In the Atherosclerosis Risk in Communities (ARIC), in a chose gathering of participants the value of vitamin D concentration below $17.2 \mathrm{ng} / \mathrm{ml}$ was associated with a higher risk of stroke [25] and a higher risk of CAD [26].

Goleniewska et al. conducted a study on 130 patients with ST-elevation myocardial infarction (STEMI), they identified vitamin D level as an independent predictor of multivessel $\mathrm{CAD}$ at multiple logistic regression analysis [27]. Another study evaluated 101 Israeli patients undergoing elective coronary arteriography by Shor et al. showed that there was a correlation between low vitamin D level and severe CAD after adjustment for confounders such as sex, age, body mass index, ethnicity and active tobacco smoking [28]. Syal et al. studied 100 Indian patients undergoing coronary angiography and reported that Indian patients with angiographically documented CAD more commonly have vitamin D deficiency. Also, a significantly higher incidence of two- and three-vessel disease was found in patients with vitamin D deficiency compared to patients with a normal vitamin D level [29]. Besides, Gondim et al. inspected 166 patients with type 2 diabetes determined to have STEMI and observed a higher percentage of vitamin D deficiency compared to patients without STEMI. Also, a higher incidence of multivessel disease was observed in diabetic patients with vitamin D deficiency compared to nondiabetic patients [30]. Shanker et al. studied 287 Indian patients with CAD and reported that vitamin D levels were significantly lower than in matched healthy controls, and patients with low vitamin D levels having a greater risk of CAD than those with higher vitamin D levels [31]. Also, Verdoia M, et al. conducted a study on 1811 patients undergoing coronary angiography and demonstrated that lower levels of vitamin D were associated with an increased prevalence and severity of CAD in females. While in male patients, vitamin D levels were independently related to the prevalence of $\mathrm{CAD}$, but not related to the severity of CAD [32].

We identified a significant association between vitamin D deficiency and diabetes and dyslipidemia that matches with the results of a cross-sectional study in 3788 Chinese subjects conducted by Jiang X, et al. who concluded that Vitamin D deficiency was significantly associated with dyslipidemia and serum vitamin D was inversely correlated with LDL cholesterol and triglycerides levels, and positively correlated with HDL cholesterol level [33]. Also, Jorde R, et al. showed that there was a significant increase in serum TC, HDL-C, and LDL-C, and a significant decrease in serum LDL$\mathrm{C} / \mathrm{HDL}-\mathrm{C}$ ratio and TAG across increasing serum $25(\mathrm{OH}) \mathrm{D}$ quartiles [34]. Another study conducted on 150 Indian patients by Chaudhuri JR, et al. showed that vitamin D deficiency was found in $39.3 \%$ of patients and significantly associated with dyslipidemia and mean serum glucose [35].
Ge H, et al., conducted a study with 1191 participants and reported that serum $25(\mathrm{OH}) \mathrm{D} 3$ deficiency was associated with dyslipidemia [36]. Alhewishel M A, et al. 2020 investigated the association between diabetes and vitamin D level in Saudi Arabia and showed that $89.53 \%$ of the patients had a vitamin $\mathrm{D}$ level below the normal range and vitamin $\mathrm{D}$ level was inversely proportional to the level of fasting glucose and HbAlc [37]. Dalgård $\mathrm{C}$, et al. also, concluded that vitamin D sufficiency may protect against type 2 diabetes in elderly subjects [38].

Despite all this evidence supporting the association between vitamin $\mathrm{D}$ level and diabetes, dyslipidemia, and severity of coronary artery disease, the results of vitamin D supplementation are still controversial as regard this issue. Sugden et al. showed that vitamin D supplementation improved endothelial function in diabetics with low vitamin D levels [39]. A randomized trial conducted by Witham et al. agreed with Sugden et al. in that a single high dose of vitamin D2 improves endothelial function [39, 40]. A systematic review and meta-analysis created by $\mathrm{Wu}$ et al. concluded that vitamin D supplementation could be successful at improving glycemic control, in a chose gathering of diabetic patients, that is, with vitamin D deficiency at baseline or nonobese [41]. Other studies suggested a beneficial effect of vitamin D supplementation only in patients at risk of diabetes [42-44]. In contrast, Sokol et al. showed no change in proinflammatory cytokine levels and endothelial function in patients with CAD after repletion of cholecalciferol [45]. Similar results of a systematic review and a meta-analysis created by Elamin MB, et al. who failed to detect a statistically significant reduction in mortality and cardiovascular risks, such as stroke and myocardial infarction, after vitamin D supplementation [46].

We need more trials focusing on patients at major risk for cardiovascular diseases, and those with well-established cardiovascular diseases and severe atherosclerosis to study the actual benefit of vitamin D supplementation in those patients.

\section{Limitations of Our Study}

First, it was a cross-section observational study that only suggests a statistical association but cannot prove it. Secondly, we did not consider some confounders that may affect the results such as medications used by the participants especially, statins that may affect the severity of atherosclerosis or diet and some drugs that may affect vitamin D level. Finally, we assessed the severity of CAD depending on coronary angiography and gensini score that does not consider coronary calcifications.

\section{Conclusion}

In our study, vitamin D deficiency, diabetes, dyslipidemia, higher BMI, and ACS were statistically significant associated with severe coronary atherosclerosis. Also, diabetes and dyslipidemia were statistically significant associated with 
vitamin D deficiency. Therefore, it might be concluded that vitamin D deficiency is a significant predictor of diabetes, dyslipidemia, and severe CAD. Large randomized controlled trials are required to study the effect of vitamin D supplementation on the reduction of cardiovascular risk and severity of CAD.

\section{List of Abbreviations}

$\mathrm{ACS}=$ acute coronary syndrome

$\mathrm{BMI}=$ body mass index

$\mathrm{CAD}=$ coronary artery disease

$\mathrm{CI}=$ confidence interval

$\mathrm{CX}=$ circumflex artery

$\mathrm{LAD}=$ left anterior descending artery

$\mathrm{OR}=$ odds ratio

$\mathrm{SD}=$ standard deviation

$\mathrm{STEMI}=\mathrm{ST}$ elevation myocardial infarction.

\section{References}

[1] De Luca G., Smit JJ, Ernst N, et al. Impact of adjunctive tirofiban administration on myocardial perfusion and mortality in patients undergoing primary angioplasty for ST-segment elevation myocardial infarction. Thromb. Haemost. 2005; 93: pp. 820-823.

[2] De Luca G., Verdoia M., and Suryapranata H. Benefits from intracoronary as compared to intravenous abciximab administration for STEMI patients undergoing primary angioplasty: a meta-analysis of 8 randomized trials. Atherosclerosis 2012; 222: pp. 426-433.

[3] De Luca G., Navarese E. P., and Suryapranata H. A metaanalytic overview of thrombectomy during primary angioplasty. Int. J. Cardiol. 2013; 166: pp. 606-612.

[4] De Luca G, Dirksen MT, Spaulding C, et al. Impact of hypertension on clinical outcome in STEMI patients undergoing primary angioplasty with BMS or DES: insights from the DESERT cooperation. Int J Cardiol. 2014; 175 (1): 50-54.

[5] Zittermann A, Schleithoff SS, Koerfer R. Putting cardiovascular disease and vitamin D insufficiency into perspective. Br J Nutr. 2005; 94: 483-92.

[6] Holick MF. Vitamin D deficiency. N Engl J Med. 2007; 357: 266-81.

[7] Holick MF, Binkley NC, Bischoff-Ferrari HA, et al. Evaluation, treatment, and prevention of vitamin D deficiency: an Endocrine Society clinical practice guideline. J Clin Endocrinol Metab. 2011; 96: 1911-30.

[8] Querfeld U.: Vitamin D and inflammation. Pediatr. Nephrol. 2013; 28: pp. 605-610.

[9] Buitrago C. G., Arango N. S., and Boland R. L.: 1 $\alpha, 25(\mathrm{OH})$ 2D3-dependent modulation of Akt in proliferating and differentiating $\mathrm{C} 2 \mathrm{C} 12$ skeletal muscle cells. J. Cell. Biochem. 2012; 113: pp. 1170-1181.

[10] Li YC, Kong J, Wei M, et al. 1,25-Dihydroxyvitamin D (3) is a negative endocrine regulator of the emode-angiotensin system. J Clin Invest. 2002; 110: 229-38.

[11] Kassi E, Adamopoulos C, Basdra EK, et al. Role of vitamin D in atherosclerosis. Circulation. 2013; 128: 2517-31.

[12] Shankar A., Sabanayagam C., and Kalidindi S.: Serum 25hydroxyvitamin $\mathrm{d}$ levels and prediabetes among subjects free of diabetes. Diabetes Care 2011; 34: pp. 1114-1119.

[13] Gagnon C, Lu ZX, Magliano DJ, et al. Low serum 25hydroxyvitamin $\mathrm{D}$ is associated with increased risk of the development of the metabolic syndrome at five years: results from a national, population-based prospective study. J Clin Endocrinol Metab. 2012; 97: 1953-61.

[14] Pittas AG, Lau J, Hu FB, Dawson-Hughes B. The role of vitamin $\mathrm{D}$ and calcium in type 2 diabetes. A systematic review and meta-analysis. J Clin Endocrinol Metab. 2007; 92: $2017-$ 29.

[15] Lamendola CA, Ariel D, Feldman D, Reaven GM. Relations between obesity, insulin resistance, and 25-hydroxyvitamin D. Am J Clin Nutr. 2012; 95: 1055-105.

[16] Dziedzic EA, Przychodzeń S, Dąbrowski M. The effects of vitamin D on severity of coronary artery atherosclerosis and lipid profile of cardiac patients. Arch Med Sci. 2016; 12: 1199-206.

[17] Josko D. Updates in immunoassays: introduction. Clin Lab Sci. 2012; 25 (3): 170-172.

[18] Gensini, G. G. (1983) A More Meaningful Scoring System for Determining the Severity of Coronary Heart Disease. American Journal of Cardiology, 51, 606.

[19] Standards of Medical Care in Diabetes. Diabetes Care 2015; 38 (Suppl 1): S3.

[20] Efrain Reisin, Raymond C. Harris, Mahboob Rahman. Commentary on the 2014 BP Guidelines from the Panel Appointed to the Eighth Joint National Committee (JNC 8). J Am Soc Nephrol. 2014 Nov; 25 (11): 2419-2424.

[21] American Diabetes Association: Diagnosis and classification of diabetes mellitus. Diabetes Care 2010; 33: pp. S62-S69.

[22] Dziedzic EA, Gąsior JS, Pawłowski M, et al. Association of Vitamin D Deficiency and Degree of Coronary Artery Disease in Cardiac Patients with Type 2 Diabetes. J Diabetes Res. 2017; 2017: 3929075.

[23] Dziedzic EA, Gąsior JS, Pawłowski M, et al. Vitamin D level is associated with severity of coronary artery atherosclerosis and incidence of acute coronary syndromes in non-diabetic cardiac patients. Arch Med Sci. 2019; 15 (2): 359-368.

[24] Verdoia M, Schaffer A, Sartori C, et al. Vitamin D deficiency is independently associated with the extent of coronary artery disease. Eur J Clin Invest. 2014; 44 (7): 634-642.

[25] Schneider AL, Lutsey PL, Selvin E, et al. Vitamin D, vitamin $\mathrm{D}$ binding protein gene polymorphisms, race and risk of incident stroke: the Atherosclerosis Risk in Communities (ARIC) study. Eur J Neurol. 2015; 22: 1220-7.

[26] Michos ED, Misialek JR, Selvin E, et al. 25-hydroxyvitamin D levels, vitamin D binding protein gene polymorphisms and incident coronary heart disease among whites and blacks: the ARIC study. Atherosclerosis. 2015; 241: 12-7. 
[27] Goleniewska BM, Kacprzak M, Zielinska M. Vitamin D level and extent of coronary stenotic lesions in patients with first acute myocardial infarction. Cardiol J 2014; 21 : $18-23$.

[28] Shor R, Tirosh A, Shemesh L, et al. 25 hydroxyvitamin D levels in patients undergoing coronary artery catheterization. Eur J Intern Med. 2012; 23: 470-3.

[29] Syal SK, Kapoor A, Bhatia E, et al. Vitamin D deficiency, coronary artery disease, and endothelial dysfunction: observations from a coronary angiographic study in Indian patients. J Invasive Cardiol. 2012; 24: 385-9.

[30] Gondim F., Caribé A., Vasconcelos K. F., et al. Vitamin D deficiency is associated with severity of acute coronary syndrome in patients with type 2 diabetes and high rates of sun exposure. Clinical Medicine Insights. Endocrinology and Diabetes. 2016; 9: 37-41.

[31] Shanker J, Maitra A, Arvind P, et al. Role of vitamin D levels and vitamin $\mathrm{D}$ receptor polymorphisms in relation to coronary artery disease: the Indian atherosclerosis research study. Coron Artery Dis 2011; 22: 324-32.

[32] Verdoia M, Schaffer A, Barbieri L, et al. Impact of gender difference on vitamin D status and its relationship with the extent of coronary artery disease. Nutr Metab Cardiovasc Dis. 2015; 25 (5): 464-470.

[33] Jiang X, Peng M, Chen S, et al. Vitamin D deficiency is associated with dyslipidemia: a cross-sectional study in 3788 subjects. Curr Med Res Opin. 2019; 35 (6): 1059-1063.

[34] Jorde R, Figenschau Y, Hutchinson M, et al. High serum 25hydroxyvitamin $\mathrm{D}$ concentrations are associated with a favorable serum lipid profile. Eur J Clin Nutr. 2010; 64 (12): $1457-1464$.

[35] Chaudhuri JR, Mridula KR, Anamika A, et al. Deficiency of 25-hydroxyvitamin $\mathrm{d}$ and dyslipidemia in Indian subjects. $\mathrm{J}$ Lipids. 2013; 2013: 623420.

[36] Ge H, Sun H, Wang T, et al. The association between serum 25-hydroxyvitamin D3 concentration and serum lipids in the rural population of China. Lipids Health Dis. 2017; 16 (1): 215.
[37] Alhewishel M A, Bahgat M, Al Huwaiyshil A, et al. $25(\mathrm{OH})$ D Serum Level in Non-Diabetic and Type II Diabetic Patients: A Cross-Sectional Study. Cureus 2020; 12 (6): e8910.

[38] Dalgård C, Petersen MS, Weihe $\mathrm{P}$, et al. Vitamin D status in relation to glucose metabolism and type 2 diabetes in septuagenarians. Diabetes Care. 2011; 34 (6): 1284-1288. doi: $10.2337 / \mathrm{dc} 10-2084$.

[39] Sugden JA, Davies JI, Witham MD, et al. Vitamin D improves endothelial function in patients with type 2 diabetes mellitus and low vitamin D levels. Diabet Med 2008; 25: 320-5.

[40] Witham MD, Dove FJ, Sugden JA, et al. The effect of vitamin D replacement on markers of vascular health in stroke patients - a randomised controlled trial. Nutr Metab Cardiovasc Dis 2012; 22: 864-70.

[41] Wu C., Qiu S., Zhu X., et al. Vitamin D supplementation and glycemic control in type 2 diabetes patients: a systematic review and meta-analysis. Metabolism. 2017; 73: 67-76.

[42] Pittas A. G., Dawson-Hughes B., Sheehan P. R., et al. Rationale and design of the vitamin $\mathrm{D}$ and type 2 diabetes (D2d) study: a diabetes prevention trial. Diabetes Care. 2014; 37 (12): 3227-3234.

[43] Mitri J., Dawson-Hughes B., Hu F., Pittas A. Effects of vitamin $D$ and calcium supplementation on pancreatic $\beta$ cell function, insulin sensitivity, and glycemia in adults at high risk of diabetes: the Calcium and Vitamin D for Diabetes Mellitus $(\mathrm{CaDDM})$ randomized controlled trial. American Journal of Clinical Nutrition. 2011; 94 (2): 486-494.

[44] Pittas A. G., Harris S. S., Stark P. C., Dawson-Hughes B. The effects of calcium and vitamin D supplementation on blood glucose and markers of inflammation in nondiabetic adults. Diabetes Care. 2007; 30 (4): 980-986.

[45] Sokol SI, Srinivas V, Crandall JP, et al. The effects of vitamin $\mathrm{D}$ repletion on endothelial function and inflammation in patients with coronary artery disease. Vasc Med 2012; 17: 394-404.

[46] Elamin MB, Abu Elnour NO, Elamin KB, et al. Vitamin D and cardiovascular outcomes: a systematic review and metaanalysis. J Clin Endocrinol Metab 2011; 96: 1931-42. 